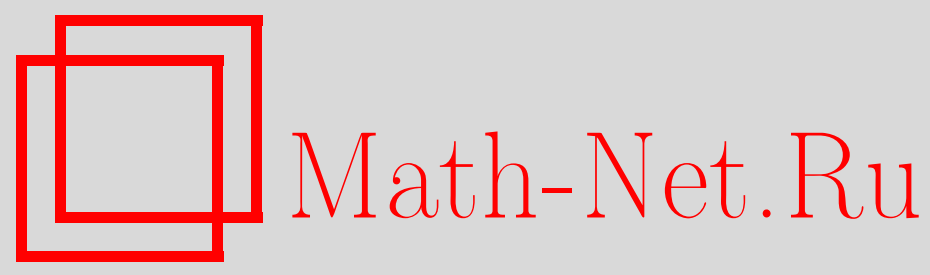

В. А. Окулов, Многомерный аналог одной теоремы Зигмунда, Матем. заметки, 1997, том 61, выпуск 5, 717-727

DOI: https://doi.org/10.4213/mzm1553

Использование Общероссийского математического портала Math-Net.Ru подразумевает, что вы прочитали и согласны с пользовательским соглашением http://www . mathnet.ru/rus/agreement

Параметры загрузки:

IP: 52.23 .180 .231

26 апреля 2023 г., 18:16:35

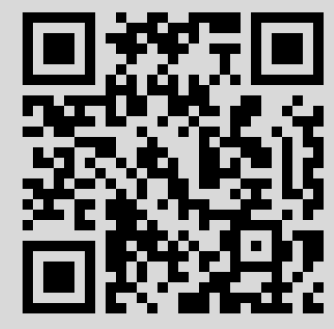




\title{
МНОГОМЕРНЫЙ АНАЛОГ ОДНОЙ ТЕОРЕМЫ ЗИГМУНДА
}

\section{В.А. Окулов}

\begin{abstract}
А. Зигмундом доказано неравенство, характеризующее зависимость модуля непрерьвности сопряженной функции от модуля непрерьвности исходной функции из пространства $2 \pi$-периодических непрерывных функций. Настоящая статья содержит оценки частных модулей непрерывности сопряженной функции многих переменных в пространстве $C$. Соответствующими примерами показана точность полученных результатов.

Библиограффия: 7 названий.
\end{abstract}

1. Введение. В настоящей работе предполагается пользоваться следующими обозначениями: $N$ - натуральное число, $M$ - множество $\{1,2, \ldots, N\}, B$ - непустое подмножество множества $M,|B|$ - количество элементов в множестве $B$. Все рассматриваемые ниже функции предполагаются $2 \pi$-периодическими по каждой переменной. Через $C\left(T^{N}\right)$, где $T=[-\pi, \pi]$, обозначается пространство $2 \pi$-периодических по каждой переменной функций, непрерывных на всем $\mathbb{R}^{N}$ с нормой

$$
\|f\|=\max _{\bar{x} \in T^{N}}|f(\bar{x})| .
$$

Пусть $f \in C\left(T^{N}\right), 1 \leqslant j \leqslant N$, и $t \in T$. Тогда для всех $\bar{x}$ положим

$$
\Delta_{j}(f, \bar{x}, t)=f\left(x_{1}, \ldots, x_{j-1}, x_{j}+t, x_{j+1}, \ldots, x_{N}\right)-f(\bar{x})
$$

и определим модуль непрерывности по $j$-й переменной равенством

$$
\omega_{j}(f, \delta)=\sup _{|t| \leqslant \delta}\left\|\Delta_{j}(f, \bar{x}, t)\right\|
$$

Если задана функция $\omega(u)$, обладающая следующими свойствами:

1) $\omega(0)=0$;

2) $\omega(u)$ не убывает на $[0, \pi]$;

3) $\omega(u)$ непрерьвна на $[0, \pi]$;

4) $\omega(\xi+\eta) \leqslant \omega(\xi)+\omega(\eta)$ при $0 \leqslant \xi \leqslant \xi+\eta \leqslant \pi$,

Работа выполнена при финансовой поддержке Российского фонда фундаментальных исследований, грант № 93-01-00240. 
то будем назьвать ее просто модулем непрерывности.

Далее, если заданы $|B|$ модулей непрерывности $\omega_{j}(\delta)(j \in B)$, то через $H\left(\omega_{j}, j \in B\right.$, $T^{N}$ ) будем обозначать класс тех $f \in C\left(T^{N}\right)$, для которых

$$
\forall j \in B: \omega_{j}(f, \delta)=O\left[\omega_{j}(\delta)\right], \quad \delta \rightarrow+0 .
$$

В частности, если $\omega_{j}(\delta)=\delta^{\alpha}$ при $j \in M, 0<\alpha \leqslant 1$ и $f \in H\left(\omega_{j}, j \in M, T^{N}\right)$, то говорят, что функция $f(\bar{x})$ удовлетворяет условию Липшииа порядка $\alpha\left(f \in \operatorname{Lip}\left(\alpha, C\left(T^{N}\right)\right)\right)$.

Теперь определим сопряженную к $f(\bar{x})$ функцию по переменным, индексы которых составляют множество $B=\left(r_{1}, \ldots, r_{k}\right)$ (или $B$-сопряженную функцию, $B \subseteq M$ ), paвенством

$$
\bar{f}_{B}(\bar{x})=\left(-\frac{1}{2 \pi}\right)^{|B|} \lim _{\varepsilon(B) \rightarrow 0} \int_{T_{\varepsilon(B)}^{|B|}} f\left(\bar{x}+\bar{s}_{B}\right)\left(\prod_{j=1}^{k} \operatorname{ctg} \frac{s r_{j}}{2} d s_{r_{j}}\right)
$$

где $\varepsilon(B)=\left(\varepsilon_{r_{1}}, \ldots, \varepsilon_{r_{k}}\right), \varepsilon_{r_{j}}>0$ при $j=1, \ldots, k$,

$$
T_{\varepsilon(B)}^{|B|}=\prod_{j=1}^{k}\left(T \backslash\left(-\varepsilon_{r_{j}}, \varepsilon_{r_{j}}\right)\right)
$$

$N$-мерньй вектор $\bar{s}_{B}$ имеет координаты

$$
\left(\bar{s}_{B}\right)_{l}= \begin{cases}s_{r_{j}} & \text { при } l=r_{j}, \text { где } j=1, \ldots, k, \\ 0 & \text { при остальных } l .\end{cases}
$$

Предел в (1) берется при независимом стремлении к нулю компонент вектора $\varepsilon(B)$, т.е. в смысле Прингсхейма.

Хорошо известно, что уже в одномерном случае непрерьвная функция может иметь разрывную сопряженную (см., например, [1, с. 554]). Более того, А. Зигмунд [2] показал, что если для некоторого модуля непрерьвности $\omega(t)$ имеет место

$$
\int_{0}^{\pi} \frac{\omega(t)}{t} d t=\infty
$$

то существует $f \in C(T)$ такая, что $\omega(f, \delta)=O[\omega(\delta)]$ при $\delta \rightarrow+0$, а сопряженная функция $\bar{f}$ даже не ограничена. В той же работе Зигмунд доказал следующую теорему.

Теорема (Зигмунд). Если $f \in C(T)$, mo

$$
\omega(\bar{f}, \delta) \leqslant A\left\{\int_{0}^{\delta} \frac{\omega(f, t)}{t} d t+\delta \int_{\delta}^{\pi} \frac{\omega(f, t)}{t^{2}} d t\right\}
$$

при $0 \leqslant \delta \leqslant \pi / 2$, әде $A-$ абсолютная константа.

В частности, из теоремы Зигмунда следует, что если интеграл (2) конечен, то $\omega(\bar{f}, \delta) \rightarrow 0$ при $\delta \rightarrow+0$, т.е. $\bar{f} \in C(T)$.

Н. К. Бари и С. Б. Стечкин [3] показали, что в теореме Зигмунда оценка модуля непрерьвности сопряженной функции является точной по порядку. 
ТеОРема (Бари, Стечкин). Пусть $\omega(t)-$ модуль непрерывности $u$

$$
\int_{0}^{\pi} \frac{\omega(t)}{t} d t<\infty
$$

Тогда существует $f \in C(T)$ такая, что $\omega(f, \delta)=\omega(\delta)$ при $0 \leqslant \delta \leqslant \pi / 2 u$

$$
\omega(\bar{f}, \delta) \geqslant \frac{1}{\pi}\left\{\int_{0}^{\delta} \frac{\omega(t)}{t} d t+\delta \int_{\delta}^{\pi} \frac{\omega(t)}{t^{2}} d t\right\}+O[\omega(\delta)]
$$

nрu $\delta \rightarrow+0$.

В настоящей работе будут доказаны многомерные аналоги вьшеуказанных теорем.

Прежде всего приведем критерий непрерьвности сопряженных функций, доказанньй автором в работе [4].

Tеорема 1. Пусть $B \subseteq M, \omega_{j}(\delta)(j \in B)-$ модули непрерывности. Тогда для того, чтобы для любой функиии $f \in H\left(\omega_{j}, j \in B, T^{N}\right)$ сопряженная функиия $\bar{f}_{B} \in C\left(T^{N}\right)$ необходимо и достаточно, чтобы

$$
\int_{[0, \pi / 2]^{|B|} \mid} \min _{j \in B} \omega_{j}\left(\delta_{j}\right) \prod_{j \in B} \frac{d \delta_{j}}{\delta_{j}}<\infty
$$

Отметим, что достаточность условия (3) в теореме 1 следует из результата Л. К. Панджикидзе [5].

Следующая теорема является аналогом теоремы Зигмунда.

Teopema 2. Пyсmb $f \in C\left(T^{N}\right), \omega_{i}(f, \delta)=\omega_{i}(\delta) n p u 0 \leqslant \delta \leqslant \pi, 1 \leqslant i \leqslant N, B \subseteq M$ и выполнено условие (3). Тогда если $k \in B$, то

$$
\omega_{k}\left(\bar{f}_{B}, \delta\right)=O\left\{\int_{[0, \pi]^{|B|}} \min \left(\delta, s_{k}\right) s_{k}^{-1} \min _{i \in B} \omega_{i}\left(s_{i}\right) \prod_{i \in B} s_{i}^{-1} d s_{i}\right\}
$$

при $\delta \rightarrow+0$, a если $k \in M \backslash B$, mo

$$
\omega_{k}\left(\bar{f}_{B}, \delta\right)=O\left\{\int_{[0, \pi]^{|B|}} \min \left\{\min _{i \in B} \omega_{i}\left(s_{i}\right), \omega_{k}(\delta)\right\} \prod_{i \in B} s_{i}^{-1} d s_{i}\right\}
$$

при $\delta \rightarrow+0$.

Отметим, что приводимое ниже доказательство теоремы 2 с небольшими изменениями переносится на случай, когда $f \in L\left(T^{N}\right)$ и рассматриваются частные интегральные модули непрерьвности функций.

Оценки модулей непрерьвности сопряженной функции в теореме 2 являются точными. Сформулируем это утверждение в виде теоремы. 
Tеорема 3. Пусть $B \subseteq M, a \omega_{i}(\delta)(1 \leqslant i \leqslant N)-$ модули непрерывности. Тогда существуют функиии $f, g \in C\left(T^{N}\right)$ такие, что

$$
\omega_{i}(f, \delta) \leqslant \omega_{i}(\delta) \quad u \quad \omega_{i}(g, \delta) \leqslant \omega_{i}(\delta)
$$

при $0 \leqslant \delta \leqslant \pi, 1 \leqslant i \leqslant N$, но если $k \in B$, то имеет место неравенство

$$
\omega_{k}\left(\bar{f}_{B}, \delta\right) \geqslant \frac{1}{16 \pi^{|B|}} \int_{[0, \pi]^{|B|}} \min \left(\delta, s_{k}\right) s_{k}^{-1} \min _{i \in B} \omega_{i}\left(s_{i}\right) \prod_{i \in B} s_{i}^{-1} d s_{i}
$$

при $0 \leqslant \delta \leqslant \pi / 2$, а если $k \notin B($ в случае $B \neq M)$, то имеет место неравенство

$$
\omega_{k}\left(\bar{g}_{B}, \delta\right) \geqslant(N-|B|)^{-1} \pi^{-|B|} \int_{[0, \pi]^{|B|}} \min \left\{\min _{i \in B} \omega_{i}\left(s_{i}\right), \omega_{k}(\delta)\right\} \prod_{i \in B} s_{i}^{-1} d s_{i} .
$$

Отметим, что из теорем 2 и 3 вытекают оценки М. М. Лекишвили [6] модулей непрерывности сопряженных функций для класса $\operatorname{Lip}\left(\alpha, C\left(T^{N}\right)\right)$, а также аналогичные оценки для более широкого класса функций в пространстве $C\left(T^{N}\right)$, полученные автором в работе [4].

2. Доказательство теоремы 2. Предварительно докажем следующую лемму.

ЛЕмма. Пусть $g \in L(T), g(-\pi)=g(\pi)$. Тогда при $0 \leqslant h \leqslant \pi / 2$ и для почти всех $x \in T$

$$
\Delta_{h} \bar{g}(x)=-\frac{1}{\pi} \int_{0}^{\pi} \Delta_{(s+h) / 2} \Delta_{|(s-h) / 2|} g(x) \frac{\sin h}{|\cos s-\cos h|} d s,
$$

$2 \partial e$

$$
\Delta_{h} g(x)=g(x+h)-g(x-h),
$$

а интеграл понимается как

$$
\lim _{\varepsilon \rightarrow+0}\left\{\int_{0}^{h-\varepsilon}+\int_{h+\varepsilon}^{\pi}\right\}
$$

ДоКАЗАТЕЛЬСТВО. По определению сопряженной функции

$$
(-2 \pi) \bar{g}(x+h)=\int_{T}[g(x+h+s)-g(x+h)] \operatorname{ctg} \frac{s}{2} d s .
$$

Далее, воспользовавшись $2 \pi$-периодичностью подьнтегральной функции, получаем

$$
\begin{aligned}
(-2 \pi) \bar{g}(x+h)= & \left\{\int_{0}^{\pi}+\int_{-\pi}^{0}\right\}[g(x+s)-g(x+h)] \operatorname{ctg} \frac{s-h}{2} d s \\
= & \int_{0}^{\pi}[g(x+s)-g(x+h)] \operatorname{ctg} \frac{s-h}{2} d s \\
& +\int_{-\pi}^{0} g(x+s) \operatorname{ctg} \frac{s-h}{2} d s+g(x+h) \int_{0}^{\pi} \operatorname{ctg} \frac{s+h}{2} d s .
\end{aligned}
$$


Аналогично получаем, что

$$
\begin{aligned}
(-2 \pi) \bar{g}(x-h)= & \int_{0}^{\pi} g(x+s) \operatorname{ctg} \frac{s+h}{2} d s+\int_{-\pi}^{0} g(x-h) \operatorname{ctg} \frac{s-h}{2} d s \\
& +\int_{-\pi}^{0}[g(x+s)-g(x-h)] \operatorname{ctg} \frac{s+h}{2} d s .
\end{aligned}
$$

Поэтому

$$
\begin{aligned}
(-2 \pi) \Delta_{h} \bar{g}(x)= & \int_{0}^{\pi}[g(x+s)-g(x+h)]\left\{\operatorname{ctg} \frac{s-h}{2}-\operatorname{ctg} \frac{s+h}{2}\right\} d s \\
& -\int_{-\pi}^{0}[g(x+s)-g(x-h)]\left\{\operatorname{ctg} \frac{s+h}{2}-\operatorname{ctg} \frac{s-h}{2}\right\} d s \\
= & \int_{0}^{\pi}[g(x+s)-g(x+h)+g(x-s)-g(x-h)] \frac{2 \sin h}{\cos h-\cos s} d s \\
= & 2 \int_{0}^{\pi} \Delta_{(s+h) / 2} \Delta_{(s-h) / 2} g(x) \frac{\sin h}{\cos h-\cos s} d s .
\end{aligned}
$$

Так как $\Delta_{t} g(x)=-\Delta_{-t} g(x)$ и при $0 \leqslant s \leqslant \pi$ знаки у $(s-h) / 2$ и у $\cos h-\cos s$ совпадают, то из последнего равенства следует, что

$$
(-\pi) \Delta_{h} \bar{g}(x)=\int_{0}^{\pi} \Delta_{(s+h) / 2} \Delta_{|(s-h) / 2|} g(x) \frac{\sin h}{|\cos h-\cos s|} d s .
$$

Лемма доказана.

ДОКАЗАТЕЛЬСТВО ТЕОРЕМЫ 2. Не ограничивая общности, предположим, что $B=$ $\{1, \ldots, n\}$, где $1 \leqslant n \leqslant N$. Пусть будет $\bar{f} \equiv \bar{f}_{B}$, а $e_{i}-i$-й единичньй базисньй вектор в $\mathbb{R}^{N}$.

Для $f \in C\left(T^{N}\right), \bar{x}, \bar{h} \in \mathbb{R}^{N}$ положим

$$
\Delta_{\bar{h}} f(\bar{x})=f(\bar{x}+\bar{h})-f(\bar{x}-\bar{h}),
$$

причем здесь учитывается $2 \pi$-периодичность функции $f(\bar{x})$ по каждому переменному.

По определению сопряженной функции

$$
\begin{aligned}
(-2 \pi)^{n} \bar{f}(\bar{x}) & =\int_{T^{n}} f\left(\bar{x}+\sum_{i=1}^{n} s_{i} e_{i}\right) \prod_{i=1}^{n} \operatorname{ctg} \frac{s_{i}}{2} d s_{i} \\
& =\int_{[0, \pi]^{n}} \Delta_{s_{1} e_{1}} \cdots \Delta_{s_{n}} e_{n} f(\bar{x}) \prod_{i=1}^{n} \operatorname{ctg} \frac{s_{i}}{2} d s_{i} .
\end{aligned}
$$

Напомним, что интеграл здесь понимается в смысле Прингсхейма.

При $0<h \leqslant \pi / 2$ из равенства (8) вытекает, что

$$
(2 \pi)^{n}\left|\Delta_{h e_{k}} f(\bar{x})\right|=\left|\int_{[0, \pi]^{n}} \Delta_{h e_{k}} \Delta_{s_{1} e_{1}} \cdots \Delta_{s_{n} e_{n}} f(\bar{x}) \prod_{i=1}^{n} \operatorname{ctg} \frac{s_{i}}{2} d s_{i}\right| .
$$


Легко видеть, что при $\bar{a}_{i} \in \mathbb{R}^{N}$, где $i=1,2, \ldots, m$,

$$
\left|\Delta_{\bar{a}_{1}} \cdots \Delta_{\bar{a}_{m}} f(\bar{x})\right| \leqslant 2^{m-1} \min _{1 \leqslant i \leqslant m}\left\{\sup _{\bar{x} \in T^{N}}\left|\Delta_{\bar{a}_{i}} f(\bar{x})\right|\right\} .
$$

Теперь определим функцию $\alpha: T \rightarrow \mathbb{R}$ следующим образом:

$$
\alpha(x)= \begin{cases}0 & \text { при } x \in[-\pi, 0), \\ x & \text { при } x \in[0, \pi / 2], \\ \pi-x & \text { при } x \in(\pi / 2, \pi] .\end{cases}
$$

Так как функция $f$ по условию $2 \pi$-периодическая по всем переменным, то

$$
\Delta_{s e_{i}} f(\bar{x})=-\Delta_{(\pi-s) e_{i}} f\left(\bar{x}+\pi e_{i}\right)
$$

при $1 \leqslant i \leqslant N, s \in \mathbb{R}$. Поэтому

$$
\sup _{\bar{x} \in T^{N}}\left|\Delta_{s e_{i}} f(\bar{x})\right| \leqslant \omega_{i}(2 \alpha(s))
$$

при $1 \leqslant i \leqslant N, 0 \leqslant s \leqslant \pi$.

Неравенства (10) и (11) вместе с равенством (9) дают следующую оценку

$$
\omega_{k}(\bar{f}, 2 h) \leqslant(2 \pi)^{-n} \int_{[0, \pi]^{n}} 2^{n} \min \left\{\min _{1 \leqslant i \leqslant n} \omega_{i}\left(2 \alpha\left(s_{i}\right)\right), \omega_{k}(2 h)\right\} \prod_{i=1}^{n} \operatorname{ctg} \frac{s_{i}}{2} d s_{i} .
$$

Поскольку функция $\alpha(x)$ симметрична относительно $\pi / 2$ на отрезке $[0, \pi]$, то из неравенства (12) следует

$$
\omega_{k}(\bar{f}, 2 h) \leqslant\left(\frac{2}{\pi}\right)^{n} \int_{[0, \pi / 2]^{n}} \min \left\{\min _{1 \leqslant i \leqslant n} \omega_{i}\left(2 s_{i}\right), \omega_{k}(2 h)\right\} \prod_{i=1}^{n} \frac{d s_{i}}{\sin s_{i}} .
$$

Теперь для получения соотношения (5) остается применить неравенство $\sin x \geqslant 2 x / \pi$ при $0 \leqslant x \leqslant \pi / 2$ и сделать тривиальную замену переменных, причем стоящий там интеграл существует уже в смысле Лебега и конечен в силу условия (3).

При доказательстве соотношения (4) предположим, не ограничивая общности, что $k=n$.

Согласно лемме

$$
\begin{aligned}
\Delta_{h e_{n}} \bar{f}(\bar{x}) & =\Delta_{h e_{n}}{\overline{\left(\bar{f}_{\{1, \ldots, n-1\}}\right)}}_{\{n\}}(\bar{x}) \\
& =-\frac{1}{\pi} \int_{0}^{\pi} \Delta_{\left(\left(s_{n}+h\right) / 2\right) e_{n}} \Delta_{\left|\left(s_{n}-h\right) / 2\right| e_{n}} \bar{f}_{\{1, \ldots, n-1\}}(\bar{x}) \frac{\sin h}{\left|\cos s_{n}-\cos h\right|} d s_{n} .
\end{aligned}
$$

Следовательно, учитывая равенство (8), получим

$$
\begin{aligned}
\Delta_{h e_{n}} \bar{f}(\bar{x})= & 2\left(-\frac{1}{2 \pi}\right)^{n} \int_{[0, \pi]^{n}} \Delta_{s_{1} e_{1}} \cdots \Delta_{s_{n-1} e_{n-1}} \Delta_{\left(\left(s_{n}+h\right) / 2\right) e_{n}} \Delta_{\left(\left(s_{n}-h\right) / 2\right) e_{n}} f(\bar{x}) \\
& \times \frac{\sin h d s_{n}}{\left|\cos s_{n}-\cos h\right|} \prod_{i=1}^{n-1} \operatorname{ctg} \frac{s_{i}}{2} d s_{i}
\end{aligned}
$$


Используя рассуждения, аналогичные тем, с помощью которых из равенства (9) была выведена оценка (12), из равенства (13) получаем

$$
\begin{aligned}
\omega_{n}(\bar{f}, 2 h) \leqslant & 2 \pi^{-n} \int_{[0, \pi]^{n}} \min \left\{\min _{1 \leqslant i \leqslant n-1} \omega_{i}\left(2 \alpha\left(s_{i}\right)\right), \omega_{n}\left(2 \alpha\left(\left|\frac{s_{n}-h}{2}\right|\right)\right)\right\} \\
& \times \frac{\sin h d s_{n}}{\left|\cos s_{n}-\cos h\right|} \prod_{i=1}^{n-1} \operatorname{ctg} \frac{s_{i}}{2} d s_{i} .
\end{aligned}
$$

Воспользовавшись симметричностью функции $\alpha(x)$ относительно $\pi / 2$ на отрезке $[0, \pi]$, получаем

$$
\begin{aligned}
2^{-n} \pi^{n} \omega_{n}(\bar{f}, 2 h) \leqslant & \left\{\int_{0}^{h}+\int_{h}^{2 h}+\int_{2 h}^{\pi}\right\} \frac{\sin h d s_{n}}{\left|\cos s_{n}-\cos h\right|} \\
& \times \int_{[0, \pi / 2]^{n-1}} \min \left\{\min _{1 \leqslant i \leqslant n-1} \omega_{i}\left(2 s_{i}\right), \omega_{n}\left(\left|s_{n}-h\right|\right)\right\} \prod_{i=1}^{n-1} \frac{d s_{i}}{\sin s_{i}} \\
= & \sum_{i=1}^{3} I_{i}(h) .
\end{aligned}
$$

Оценим интегралы $I_{i}(h)(i=1,2,3)$ по отдельности. Положим

$$
J\left(s_{n}\right)=\int_{[0, \pi / 2]^{n-1}} \min _{1 \leqslant i \leqslant n} \omega_{i}\left(2 s_{i}\right) \prod_{i=1}^{n-1} \frac{d s_{i}}{\sin s_{i}}
$$

В интеграле $I_{1}(h)$ сделаем замену $2 s_{n}^{\prime}=h-s_{n}$. Опуская штрих при $s_{n}^{\prime}$, получим

$$
I_{1}(h)=\int_{0}^{h / 2} \frac{\sin h}{\cos \left(h-2 s_{n}\right)-\cos h} J\left(s_{n}\right) d s_{n} .
$$

В интегралах $I_{2}(h)$ и $I_{3}(h)$ сделаем замену $2 s_{n}^{\prime}=s_{n}-h$.

Получаем

$$
I_{2}(h)+I_{3}(h)=\left\{\int_{0}^{h / 2}+\int_{h / 2}^{(\pi-h) / 2}\right\} \frac{\sin h}{\cos h-\cos \left(2 s_{n}+h\right)} J\left(s_{n}\right) d s_{n} .
$$

Теперь сложим $I_{1}(h)$ (формула $\left.(15)\right)$ и $I_{2}(h)$, учитьвая, что

$$
\frac{1}{\cos h-\cos \left(2 s_{n}+h\right)}+\frac{1}{\cos \left(h-2 s_{n}\right)-\cos h}=\frac{\sin h \operatorname{ctg} s_{n}}{\sin \left(s_{n}+h\right) \sin \left(h-s_{n}\right)} .
$$

В итоге преобразованное неравенство (14) запишется следующим образом

$$
\begin{aligned}
2^{-n} \pi^{n} \omega_{n}(\bar{f}, 2 h) \leqslant & \int_{0}^{h / 2} \frac{\sin ^{2} h \operatorname{ctg} s_{n}}{\sin \left(s_{n}+h\right) \sin \left(h-s_{n}\right)} J\left(s_{n}\right) d s_{n} \\
& +\int_{h / 2}^{(\pi-h) / 2} \frac{\sin h}{2 \sin s_{n} \sin \left(s_{n}+h\right)} J\left(s_{n}\right) d s_{n} .
\end{aligned}
$$


При $0 \leqslant s \leqslant h / 2 \leqslant \pi / 4$, используя неравенства

$\sin h \leqslant h, \quad \frac{2 \sqrt{2}}{3 \pi}(s+h) \leqslant \sin (s+h), \quad \frac{2}{\pi}(s-h) \leqslant \sin (h-s), \quad \operatorname{ctg} s \leqslant s^{-1}$, получаем

$$
\frac{\sin ^{2} h \operatorname{ctg} s}{\sin (s+h) \sin (h-s)} \leqslant \frac{3 \pi^{2}}{4 \sqrt{2}} \frac{h^{2}}{(s+h)(h-s) s} \leqslant \frac{11}{s} .
$$

Далее, при $0 \leqslant h / 2 \leqslant s \leqslant(\pi-h) / 2$

$$
\frac{\sin h}{2 \sin s \sin (s+h)} \leqslant \frac{h}{2 \cdot 2 / \pi \cdot s(2 \sqrt{2})(s+h) /(3 \pi)} \leqslant \frac{3 h}{s^{2}} .
$$

Подставляя оценки (17) и (18) в неравенство (16), получаем

$$
\omega_{n}(\bar{f}, 2 h)=O\left\{\int_{0}^{(\pi-h) / 2} d s_{n} \int_{[0, \pi / 2]^{n-1}} \frac{\min \left(h / 2, s_{n}\right)}{s_{n}^{2}} \min _{1 \leqslant i \leqslant n} \omega_{i}\left(2 s_{i}\right) \prod_{i=1}^{n-1} \frac{d s_{i}}{\sin s_{i}}\right\} .
$$

В силу неравенства $\sin s \geqslant 2 s / \pi(0 \leqslant s \leqslant \pi / 2)$ и неотрицательности подынтегральной функции, получаем

$$
\omega_{n}(\bar{f}, h) \leqslant \omega_{n}(\bar{f}, 4 h)=O\left\{\int_{[0, \pi]^{n}} \frac{\min \left(h, s_{n}\right)}{s_{n}} \min _{1 \leqslant i \leqslant n} \omega_{i}\left(s_{i}\right) \prod_{i=1}^{n} \frac{d s_{i}}{s_{i}}\right\} .
$$

Интеграл здесь существует в смысле Лебега и конечен, что вытекает из условия (3). Таким образом, соотношение (4) и вместе с ним теорема 2 доказаны.

3. Доказательство теоремы 3. Без ограничения общности считаем, что сопряжение ведется по первым $n(1 \leqslant n \leqslant N)$ переменньм $\left(\bar{f} \equiv \bar{f}_{\{1, \ldots, n\}}\right)$.

Рассмотрим функцию

$$
f(\bar{x})=\frac{1}{2} \min _{1 \leqslant i \leqslant n} \omega_{i}\left(2 \alpha\left(x_{i}\right)\right)
$$

при $\bar{x} \in T^{N}$ и где $\alpha(x)$ - функция, определенная в п. 2.

Заметим, что $\omega(\alpha, \delta) \leqslant 2 \delta$ и (см. [7]) $\omega\left(\omega_{i}, \delta\right)=\omega_{i}(\delta), 1 \leqslant i \leqslant n$, поэтому

$$
\omega\left(\omega_{i} \circ 2 \alpha, \delta\right) \leqslant 2 \omega_{i}(\delta), \quad 1 \leqslant i \leqslant n .
$$

Отсюда легко показать, что при $1 \leqslant i \leqslant n$ имеем $\omega_{i}(f, \delta) \leqslant \omega_{i}(\delta)$. Кроме того, отметим, что при $n \leqslant i \leqslant N$ (в случае $n \neq N) \omega_{i}(f, \delta) \equiv 0$. Покажем теперь, что функция $f$ удовлетворяет неравенству (6) при $k=n$.

Пусть $0 \leqslant \delta \leqslant \pi / 2$. По определению сопряженной функции

$$
\bar{f}\left(-2 \delta e_{n}\right)=\left(-\frac{1}{2 \pi}\right)^{n} \int_{T^{n}} f\left(s_{1}, \ldots, s_{n-1}, s_{n}-2 \delta, 0, \ldots, 0\right) \prod_{i=1}^{n} \operatorname{ctg} \frac{s_{i}}{2} d s_{i}
$$


Так как $f(\bar{x})=0$ при $\bar{x} \notin[0, \pi]^{n} \times[-\pi, \pi]^{N-n}$, то

$$
\begin{aligned}
\bar{f}\left(-2 \delta e_{n}\right) & =\left(-\frac{1}{2 \pi}\right)^{n} \int_{[0, \pi]^{n-1}} \int_{2 \delta}^{\pi+2 \delta} f\left(s_{1}, \ldots, s_{n-1}, s_{n}-2 \delta, 0, \ldots, 0\right) \prod_{i=1}^{n} \operatorname{ctg} \frac{s_{i}}{2} d s_{i} \\
& =\left(-\frac{1}{2 \pi}\right)^{n} \int_{[0, \pi]^{n}} f\left(s_{1}, \ldots, s_{n}, 0, \ldots, 0\right) \operatorname{ctg} \frac{s_{n}+2 \delta}{2}\left(\prod_{i=1}^{n-1} \operatorname{ctg} \frac{s_{i}}{2} d s_{i}\right) d s_{n}
\end{aligned}
$$

По определению функции $f$ и в силу симметричности функции $\alpha$ относительно $\pi / 2$ на отрезке $[0, \pi]$ имеем

$$
\begin{aligned}
f\left(-2 \delta e_{n}\right)= & \left(-\frac{1}{2 \pi}\right)^{n} \int_{[0, \pi / 2]^{n}} \frac{1}{2} \min _{1 \leqslant i \leqslant n} \omega_{i}\left(2 s_{i}\right)\left\{\operatorname{ctg} \frac{s_{n}+2 \delta}{2}+\operatorname{ctg} \frac{\pi-s_{n}+2 \delta}{2}\right\} d s_{n} \\
& \times \prod_{i=1}^{n-1}\left(\operatorname{ctg} \frac{s_{i}}{2}+\operatorname{ctg} \frac{\pi-s_{i}}{2}\right) d s_{i} .
\end{aligned}
$$

Учитывая, что

$$
\operatorname{ctg} \frac{s+2 \delta}{2}+\operatorname{ctg} \frac{\pi-s+2 \delta}{2}=\frac{2 \cos 2 \delta}{\sin s+\sin 2 \delta}
$$

получаем

$$
\bar{f}(0, \ldots, 0)-\bar{f}\left(-2 \delta e_{n}\right)=\frac{1}{2}\left(-\frac{1}{\pi}\right)^{n} \int_{[0, \pi / 2]^{n}} \min _{1 \leqslant i \leqslant n} \omega_{i}\left(2 s_{i}\right)\left(\prod_{i=1}^{n-1} \frac{d s_{i}}{\sin s_{i}}\right) \eta\left(s_{n}, \delta\right) d s_{n}
$$

где

$$
\eta(s, \delta)=\frac{1}{\sin s}-\frac{\cos 2 \delta}{\sin s+\sin 2 \delta}
$$

Следовательно,

$$
\omega_{n}(\bar{f}, 2 \delta) \geqslant \frac{1}{4 \pi^{n}} \int_{[0, \pi]^{n}} \min _{1 \leqslant i \leqslant n} \omega_{i}\left(s_{i}\right)\left(\prod_{i=1}^{n-1} \frac{d s_{i}}{s_{i}}\right) \eta\left(\frac{s_{n}}{2}, \delta\right) d s_{n}
$$

Обозначим

$$
\nu(s, \delta)=\frac{\cos 2 \delta \sin s}{\sin s+\sin 2 \delta} .
$$

При $0 \leqslant \delta \leqslant \pi / 4,0 \leqslant s \leqslant \delta / 2$ имеем

$$
\nu(s, \delta)=\frac{\cos 2 \delta \sin s}{2 \sin ((s+2 \delta) / 2) \cos ((2 \delta-s) / 2)} \leqslant \frac{\sin s}{2 \sin (5 s / 2)} \leqslant \frac{s}{2 \cdot 2 / \pi \cdot 5 s / 2} \leqslant \frac{\pi}{10}<\frac{1}{2},
$$

а при $\pi / 4 \leqslant \delta \leqslant \pi / 2,0 \leqslant s \leqslant \delta / 2$ функция $\nu(s, \delta)$ не положительна. Следовательно, при $0 \leqslant \delta \leqslant \pi / 2,0 \leqslant s \leqslant \delta / 2$ получаем

$$
\eta(s, \delta)=\frac{1}{\sin s}(1-\nu(s, \delta)) \geqslant \frac{1}{2 s}
$$


Далее, при $0 \leqslant \delta \leqslant \pi / 2, \delta / 2 \leqslant s \leqslant \pi / 2$ вьполнены неравенства

$$
\begin{aligned}
\eta(s, \delta) & =\frac{\sin s(1-\cos 2 \delta)+\sin 2 \delta}{\sin s(\sin s+\sin 2 \delta)} \geqslant \frac{2 \sin \delta(\sin s \sin \delta+\cos \delta)}{5 \sin ^{2} s} \\
& \geqslant \frac{2 \delta}{\pi} \frac{2 \sin s \sin \delta+2 \cos \delta}{5 s^{2}} \geqslant \frac{2 \delta}{5 \pi} \frac{\sin ^{2} \delta+2 \cos \delta}{s^{2}} \geqslant \frac{\delta}{8 s^{2}}
\end{aligned}
$$

Объединяя оценки $(20)$ и $(21)$, получаем

$$
\eta(s, \delta) \geqslant \frac{\min (s, \delta / 2)}{4 s^{2}}
$$

поэтому

$$
\eta\left(\frac{s}{2}, \delta\right) \geqslant \frac{\min (s, \delta)}{2 s^{2}}
$$

при $0 \leqslant \delta, s / 2 \leqslant \pi / 2$. Подставим последнюю оценку в неравенство (19). Находим, что

$$
\omega_{n}(\bar{f}, 2 \delta) \geqslant \frac{1}{8 \pi^{n}} \int_{[0, \pi]^{n}} \min _{1 \leqslant i \leqslant n} \omega_{i}\left(s_{i}\right) \frac{\min \left(\delta, s_{n}\right)}{s_{n}} \prod_{i=1}^{n} \frac{d s_{i}}{s_{i}}
$$

Остается заметить, что

$$
\omega_{n}(\bar{f}, \delta) \geqslant \frac{1}{2} \omega_{n}(\bar{f}, 2 \delta)
$$

и неравенство (6) доказано для $k=n$. При $n>1$ для других $k \in B=\{1, \ldots, n\}$ доказательство неравенства (6) совершенно аналогично. Таким образом, функция $f$ удовлетворяет условиям теоремы 3 .

Если $n<N$, то возможен случай, когда $k \notin B$, т.е. $n<k \leqslant N$. Для таких значений $k$ рассмотрим следуюшие $2 \pi$-периодические непрерывные на $T^{N}$ функции

$$
g^{(k)}(\bar{x})=\frac{1}{2} \min \left\{\min _{1 \leqslant i \leqslant n} \omega_{i}\left(2 \alpha\left(x_{i}\right)\right), \omega_{k}\left(\alpha\left(x_{k}\right)\right)\right\} .
$$

Теми же рассуждениями, что и выше, для функции $f$ можно показать, что $\omega_{i}\left(g^{(k)}, \delta\right) \leqslant$ $\omega_{i}(\delta)$ при $1 \leqslant i \leqslant N, n<k \leqslant N$. Оценим снизу $\omega_{k}\left(g^{(k)}, \delta\right)$ при $n<k \leqslant N$, причем, не ограничивая общности, рассмотрим случай, когда $k=N$.

Пользуясь определением сопряженной функции, функции $g^{(k)}(\bar{x})$ и симметричностью функции $\alpha$ относительно $\pi / 2$ на отрезке $[0, \pi]$, получаем

$$
\bar{g}^{(N)}(0, \ldots, 0, \delta)=\left(-\frac{1}{\pi}\right)^{n} \int_{[0, \pi / 2]^{n}} \min \left\{\min _{1 \leqslant i \leqslant n} \omega_{i}\left(2 s_{i}\right) \omega_{N}(\delta)\right\} \prod_{i=1}^{n} \frac{d s_{i}}{\sin s_{i}}
$$

Заметим, что $\bar{g}^{(N)}(0, \ldots, 0)=0$, поэтому

$$
\omega_{N}\left(\bar{g}^{(N)}, \delta\right) \geqslant \pi^{-n} \int_{[0, \pi]^{n}} \min \left\{\min _{1 \leqslant i \leqslant n} \omega_{i}\left(s_{i}\right), \omega_{N}(\delta)\right\} \prod_{i=1}^{n} \frac{d s_{i}}{s_{i}}
$$


Положим теперь

$$
g(\bar{x})=\frac{1}{N-n} \sum_{k=n+1}^{N} g^{(k)}(\bar{x}) .
$$

Легко видеть, что из неравенств $\omega_{i}\left(g^{(k)}, \delta\right) \leqslant \omega_{i}(\delta)$, где $1 \leqslant i \leqslant N, n<k \leqslant N$, вытекают неравенства $\omega_{i}(g, \delta) \leqslant \omega_{i}(\delta)$ при $1 \leqslant i \leqslant N$. Кроме того,

$$
\omega_{k}(\bar{g}, \delta)=\frac{1}{N-n} \omega_{k}\left(\bar{g}^{(k)}, \delta\right)
$$

при $n<k \leqslant N, 0 \leqslant \delta \leqslant \pi$. Поэтому из оценки (22) (и аналогичных ей оценок в случае $N-n>1)$ вытекают неравенства (7).

Теорема 3 полностью доказана.

В заключение автор выражает благодарность своему научному руководителю М.И. Дьяченко за постановку задачи и внимание к работе.

Московский государственный университет

Поступило

им. М.В. Ломоносова

\section{СПИСОК ЦИТИРОВАННОЙ ЛИТЕРАТУРЫ}

[1] Бари Н. К. Тригонометрические ряды. М.: Физматгиз, 1961.

[2] Zygmund A. O module ciaglosci sumy szeregu sprzezonego z szeregiem Fouriera // Prace Mat.-Fiz. 1924. V. 33. P. 125-132.

[3] Бари Н. К., Стечкин С. Б. Наилучшие приближения и дифференциальные свойства двух сопряженных функций // Тр. ММО 1956. Т. 5. С. 483-522.

[4] Окулов В. А. Многомерный аналог одной теоремы Привалова // Матем. сб. 1995. Т. 186. №2. C. 93-104.

[5] Панджикидзе Л.К. Сходимость кратных сопряженных тригонометрических рядов в пространстве $C\left(\mathbb{R}_{n}\right)$ и непрерывность сопряженных функций многих переменных // Сообщ. АН ГССР. 1988. Т. 132. №3. С. 481-483.

[6] Лекишвили M. M. О сопряженных функциях многих переменных в классе Lip $\alpha$ // Матем. заметки. 1978. Т. 28. №3. С. 261-272.

[7] Никольский С.M. Ряды Фурье функций с данным модулем непрерывности // Докл. AH CCCP. 1946. T. 52. C. 191-194. 